\title{
A Cost-Effective, Single-Phase Line-Interactive UPS System that Eliminates Inrush Current Phenomenon for Transformer-Coupled Loads
}

\author{
Syed Sabir Hussain Bukhari*, Shahid Atiq*, Thomas A. Lipo** and Byung-il Kwon ${ }^{\dagger}$
}

\begin{abstract}
Sudden voltage drops and outages frequently disturb the operation of sensitive loads for domestic, commercial, and industrial use. In some cases, these events may even impair the functioning of relevant equipment. To maintain power under such conditions, a UPS system is usually installed. Once a disturbance happens at the grid side, the line-interactive UPS system takes over the load to prevent an interruption. But, due to magnetic saturation of the transformer, a significant inrush current may occur for the transformer-coupled loads during this transition. The generation of such transient currents may in turn decrease the line voltage and activates over-current protecting devices of the system. In this work, a cost-effective, line-interactive UPS system is proposed that eliminates the inrush current phenomenon associated with transformer-coupled loads. The strategy was implemented by connecting a standard current-regulated voltage source inverter (CRVSI) to the secondary winding of the load transformer. During any transient condition at the grid side, the load current is monitored and regulated to achieve either seamless compensation of the load current or complete transferal of load from grid to the inverter. Experimental results were obtained for a prototype under all possible operating conditions so as to validate the performance of the proposed topology.
\end{abstract}

Keywords: UPS systems, Single-phase line-interactive, Inrush current elimination

\section{Introduction}

Nowadays, the reliability of electrical power has been key concern for domestic, commercial, and industrial users. Sudden voltage drops and outages, can interrupt the operation of critical load applications leading to the impairment of relevant equipment [1]. To address this issue and supply unpolluted and continuous power to sensitive loads during irregular utility power circumstances, a UPS system is usually installed [2]. The holdup time of a UPS system against instability is utterly dependent on the rating of the load and the energy storing capability of the UPS setup. On the basis of configuration and operating principles, UPS systems are mainly categorized into three major types: 1) offline, 2) online, and 3) line-interactive [3, 4]. However, line-interactive UPS systems can be further categorized into two topologies: 1) single converter-based topology and 2) double converter-based topology.

The oldest line-interactive UPS topology is the single converter-based type shown in Fig. 1(a); it comprises of a bi-directional converter coupled in parallel with the utility and load. The converter acts as a charger when the utility is

$\dagger$ Corresponding Author: Dept. of Electronic Systems Engineering, Hanyang University, Korea. (bikwon@hanyang.ac.kr)

* Dept. of Electronic Systems Engineering, Hanyang University, Korea. (sabir@hanyang.ac.kr)

** Dept. of Electrical and Computer Engineering, Florida State University, USA. (lipo@caps.fsu.edu)

Received: July 2, 2015; Accepted: December 12, 2015 in a healthy condition. However, the installation also works as an inverter to supply backup power under abnormal utility power conditions. For the isolation of load, a common approach is to install a high leakage inductance series transformer between the utility and load [4, 5]. Conversely, it increases the overall size of the system. In spite of offering low reactive power compensation, this type of the line-interactive UPS system provides the advantage of input current harmonic suppression.

One particular line-interactive UPS system that has received major consideration in the earlier few years is shown in Fig. 1(b). This apparatus consists of two converters, one connected in series with the utility and another in parallel with the load. During normal operation, standard power flow proceeds from the utility to the load. Meanwhile, the converter connected in parallel operates as a charger for the backup. However, when the utility fails, a bypass switch is activated so as to isolate the load from the utility; the power is then supplied to the load using the parallel converter from the battery. In addition to acting as a charger, this converter also enables the UPS system to perform input current harmonic suppression. The series converter serves as a voltage controller to maintain a suitable output voltage during utility voltage sags or swells. While this type of line-interactive UPS system offers relatively high efficiency when compared to other UPS topologies, it has the disadvantages of a complex control strategy and an absence of load isolation. The series 
transformer required in this type of topology also rises the total cost, size, and weight of the system.

The transition of a load from utility to the backup for both types of line-interactive UPS topologies takes a minimum of $1-5 \mathrm{msec}$. This interval mainly depends on the time required for fault detection and the operating mechanism of the bypass switches $[4,6]$. During this load transition time interval, a coupled transformer, is likely to be exposed to a faulty/distorted utility voltage, thereby producing a flux offset for the load transformer. Hence, as soon as the UPS system takes over the load and reinstates its rated voltage, the transformer flux may rise above its saturation level and generate a substantial inrush transient current. The size of this transient current can attain a peak that is 2-6 times the rated load current depending on the nature of the fault at the utility side, the load transition time interval for the UPS system, and the magnetic properties of the load transformer [1, 7]. Possible consequences of the inrush current include a decreased voltage and triggering of the over-current protecting devices of the UPS system, which could in turn lead to failure of the backup power.

Numerous strategies to decrease the magnitude of inrush current have been suggested by researchers. While such methods can also be employed to reduce the inrush current associated with a line-interactive UPS system while powering transformer-coupled loads, they possess a few disadvantages that restrict their adoption. Decreasing the inverter voltage when sensing the size of the inrush current [8] or injecting the UPS voltage at suitable instants [9] could be implemented to reduce the magnitude of the inrush current, but these steps usually increase the overall transition time interval for the UPS system. Furthermore, when compared to conventional setups, it is likely that sensitive loads adopting these steps will remain unprotected from a fault for a longer time. Resistors or reactors could be inserted during the load transition process, but this will increase the overall size of the system since large power panels are required to accommodate the resistors, reactors, and electromechanical switches. A flux offset compensation method can also be adopted to control the flux of the load transformer [1]. However, even with a complex control strategy, the phenomenon of inrush current will persist, albeit with a reduced magnitude.

The goal of this study is to eliminate the inrush current phenomenon for the transformer-coupled loads and obtain fast current injection by the inverter under any abnormal utility power condition. To achieve this objective, a costeffective single-phase line-interactive UPS system through a standard CRVSI coupled to the secondary winding of the transformer is proposed. The current control scheme employs recently developed high bandwidth algorithms designed for variable frequency motor control [3]. But, these approaches are also ideal for direct control of $\mathrm{AC}$ currents with or without a motor load. The possibility of inrush current generation is entirely eliminated via instantaneous compensation of the load current and a seamless transition of the load under any abnormal power conditions. Furthermore, the suggested single-phase UPS system is inexpensive, small in size and exhibits high efficiency along with power regulating capabilities.

\section{Basic Operating Principle}

As presented in Fig. 1(c), the suggested line-interactive UPS topology consists of a bypass switch connecting the transformer-coupled load to the utility, an inverter coupled to the secondary winding of the transformer, and a battery that can be charged by the rectifier/charger. While the configuration of the devised topology is similar to that of a typical offline UPS topology, its behavior during operation resembles that of a classic online UPS system, as it is always connected to the load and continuously monitors the load current to provide precise power regulation. However, unlike an online UPS system, the primary powering path for the load is the utility. Under normal utility power conditions, current is supplied to the load by the utility via bypass thyristors. In the event of an abnormality on the utility side, the inverter either supplies the difference between the rated load and measured utility currents or takes over the load completely. In either case, the action of the inverter occurs instantaneously. Compensation of the load current is limited from less than $1 \%$ to as much as $50 \%$ of the rated load current to avoid the flow of inverter current to the utility. Above the upper limit or in the case of a blackout, the bypass switch isolates the load from the utility and the UPS starts operating in inverter mode. Although the operation of bypass switches at the beginning of inverter mode takes $1-5 \mathrm{msec}$, the magnitude of the load current is not affected at any stage. This is due to the fact that transition of the load and compensation of the load current are achieved through the current control algorithm. In particular, the current at the load side $\left(I_{\text {Load }}\right)$ is measured and then used for comparison with the commanded reference signal, i.e., the rated load current $\left(I_{\text {Load }}{ }^{*}\right)$. Upon comparison of the measured and reference currents, an error signal is generated for the PI controller of the current regulator. A controlled signal is then sent from the PI controller to the PWM generator for the required commutation of the IGBTs, as shown in Fig. 1(d). Compensation of the load current and transition of the load is seamless, thereby eliminating the possibility of inrush current generation. In addition to these features, the proposed UPS system offers many advantages over conventional line-interactive UPS topologies.

The devised UPS system can be utilized in medium and high power sensitive load applications as a double converter-based line-interactive UPS topology. The total power capacity for the proposed UPS system is about $110 \%$ of the rated load power [4]. Since the developed system consists of a single inverter with low power capacity, it is inexpensive and has a small size and weight 


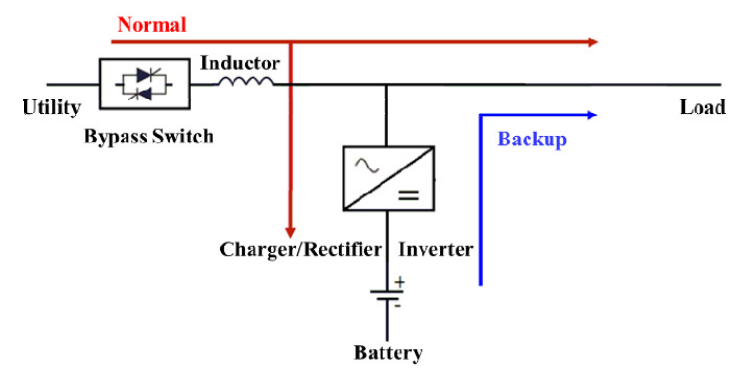

(a)

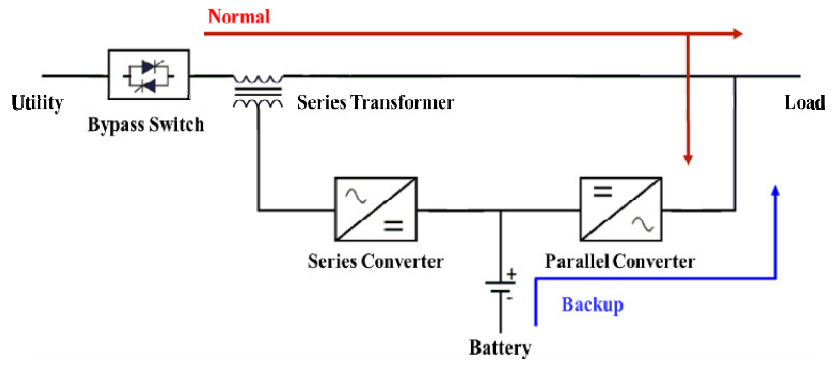

(b)

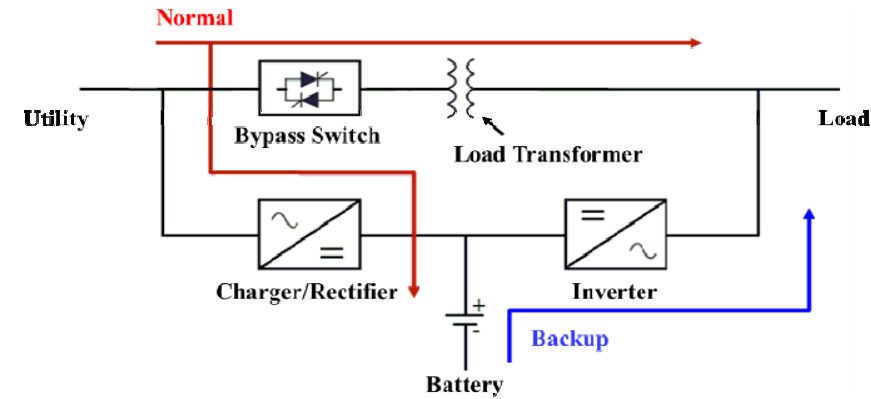

(c)

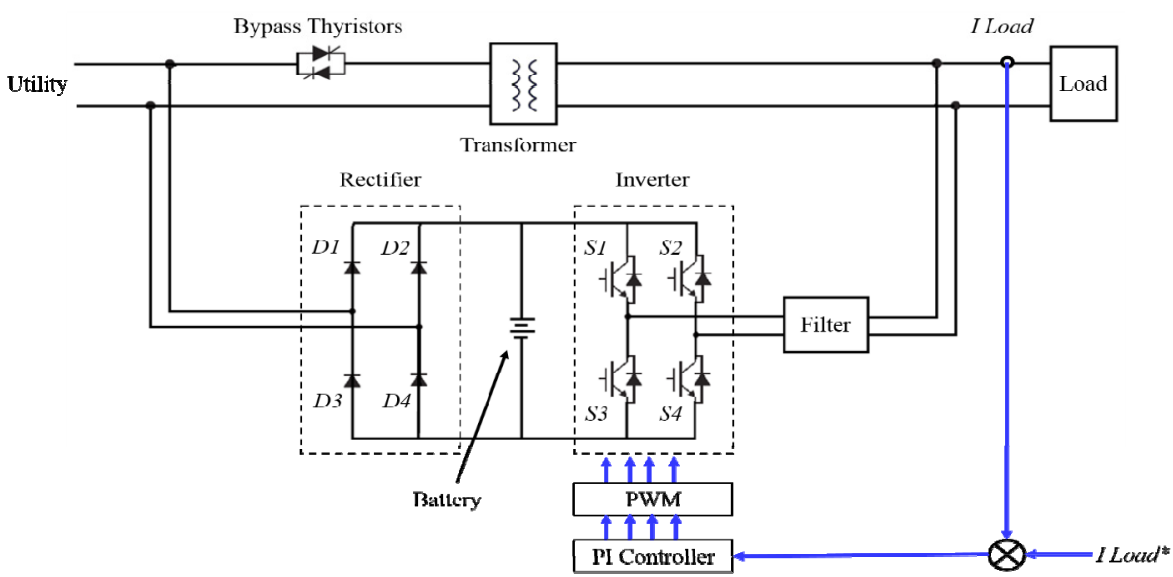

(d)

Fig. 1. Simplified diagram of (a) the single converter-based line-interactive UPS topology, (b) double converter-based lineinteractive UPS topology, (c) proposed line-interactive UPS topology, and (d) complete block diagram of the proposed line-interactive UPS topology.

Table 1. Comparative Analysis

\begin{tabular}{|c|c|c|c|}
\hline Attributes & $\begin{array}{c}\text { Single Converter-Based } \\
\text { Topology } \\
\end{array}$ & $\begin{array}{c}\text { Double Converter- } \\
\text { Based Topology }\end{array}$ & Proposed Topology \\
\hline Power Application & Low-Medium & Medium-High & Medium-High \\
\hline Power Capacity & $110 \%-120 \%$ & $120 \%$ & $110 \%$ \\
\hline Efficiency & Low & High & High \\
\hline Isolation of the Load & Better & No Effective Isolation & Best \\
\hline Power Regulation and Compensation Capabilities & No & Yes & Yes \\
\hline Cost, Size, and Weight & Low & High & Low \\
\hline Load Transition Time Interval & $1-5 \mathrm{msec}$ & $1-5 \mathrm{msec}$ & Negligible \\
\hline Inrush Current Possibility (for a Transformer-Coupled Load) & Yes & Yes & No \\
\hline
\end{tabular}

when compared to conventional double converter-based line-interactive UPS technologies. Operation of the proposed UPS system involves single power conversion, which increases its efficiency. In most of the UPS topologies, isolation of the load from the utility is achieved through the use of a transformer, which serves to increase the dimensions, mass, and price of the system. Because the proposed topology is already based on a transformer, no installation of supplementary equipment for isolation purposes is required. In addition to its power regulating 
and compensating capabilities, the proposed system employs a simple and easy control scheme that makes it superior to double converter-based line-interactive UPS architectures. A detailed comparison of the suggested and conventional line-interactive UPS topologies is given in Table 1 .

Both the configuration of the proposed topology and relevant controller design concerns will be discussed in the following sections.

\subsection{Line-commutation}

In conventional line-interactive UPS topologies, the detection of a power failure by the controller leads to activation of a bypass switch so as to isolate the load from the utility. The load is subsequently supplied from the UPS system. However, due to the natural commutation characteristics of the thyristor, this load transition takes about $4 \mathrm{msec}$. Consequently, the transformer-coupled loads are exposed to a faulty/distorted utility voltage, thereby establishing a flux offset for the transformer. The flux of the load transformer could thus be driven beyond its saturation limit, causing a significant inrush current. To study the performance of the conventional and suggested UPS systems, simulations were performed using a load of $1 \mathrm{kVA}$. A power failure has been introduced at the utility side when $t=0.175 \mathrm{sec}$, and transition of the load occurred at $t=0.179 \mathrm{sec}$. This load transition caused an inrush current of approximately $1.8 \mathrm{pu}$, which is approximately $80 \%$ greater than the steady-state rated load current. However, for the proposed UPS system, the load transition is seamless. Furthermore, the magnitude of the load current is constant and never go beyond from the prescribed limit at any instant, as presented in Fig. 2.

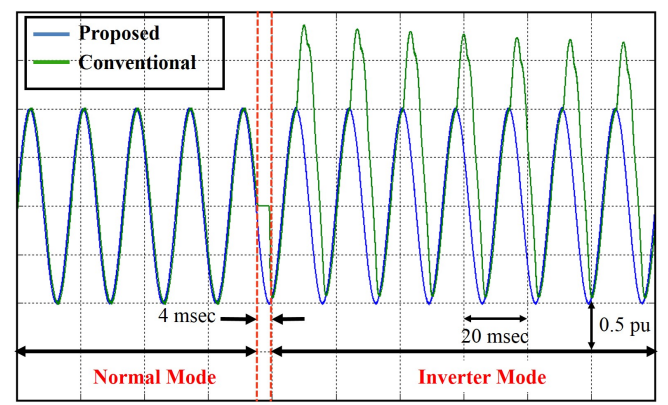

Fig. 2. Simulated load currents using both the conventional and proposed line-interactive UPS systems

\subsection{Current regulation algorithm implemented for the inverter}

The capability of the proposed cost-effective UPS system to eliminate the transient inrush current is achieved through the use of a current-regulating algorithm for the inverter. Any recently developed current control schemes can be implemented for this purpose. However, current regulation in this work is attained with a PI controller using a stationary frame of reference, as discussed in [10] since it is simple and easy to implement.

The transfer function of the proposed UPS system is given by:

$$
Y(s)=\frac{I(s) * Z(s) * G p(s)}{1+G c(s) * V_{D C} * G p(s)}+\frac{U(s) * G c(s) * V_{D C} * G p(s)}{1+G c(s) * V_{D C} * G p(s)}
$$

where $U(\mathrm{~s})$ stands for the commanded current, $Y(\mathrm{~s})$ is the inverter output current, $E(\mathrm{~s})$ is the error signal and $I(\mathrm{~s})$ is the utility input current injection. $V_{D C}$ is the forward gain of the linear amplifier which is replaced for the PWM modulator. $G_{c}(\mathrm{~s}), G_{p}(\mathrm{~s})$, and $Z(\mathrm{~s})$ are the transfer functions for the controller, plant, and load impedance respectively.

The function of the PI controller is to equate the output load current to the reference current, i.e., the rated load current, as diligently as possible.

The load impedance $Z(\mathrm{~s})$ is:

$$
Z(s)=R(s)+s L(s)
$$

where $R(\mathrm{~s})$ is the load resistance and $L(\mathrm{~s})$ is the load inductance.

For the average model representation, circuit theory yields the following $R / L$ "plant" transfer function:

$$
G_{p}(s)=\frac{1}{R(1+s T)}, T=L / R
$$

$G_{c}(\mathrm{~s})$ is given by:

$$
G_{c}(s)=K p\left[1+\frac{1}{s \tau_{r}}\right]
$$

where $\tau_{r}$ is the integrator reset time defined as the ratio of the proportional and integral gains $\left(K_{p} / K_{i}\right)$.

By substituting from Eqs. (3), and (4), the open loop forward path gain of this system is obtained as:

$$
G_{p} G_{c}(s)=G_{c}(s) * V_{D C} * G_{p}(s)=\frac{V_{D C} * K_{p} *\left(1+s \tau_{r}\right)}{R * \tau_{r} * s *(1+s T)}
$$

Like other control schemes, operation of the implemented current control system is influenced by transport and sampling delays due to the PWM process and the digital control system, respectively. The total transport and sampling delays for the implemented current regulator are about $75 \%$ of the carrier period delay, as discussed in [11].

The control loop delays can be modeled according to Ztransform theory. A zero-order-hold element is used to represent the sampling delay, while a $1 / Z$ block is 


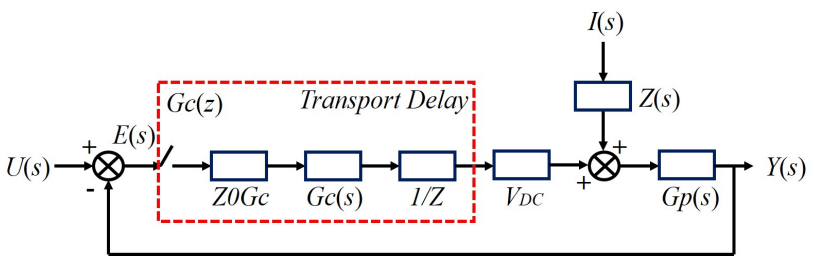

(a)

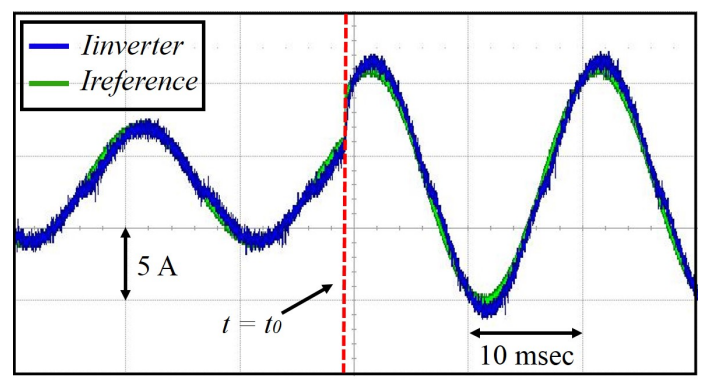

(b)

Fig. 3. (a) Average value model block diagram representation, and (b) experimental response of the current regulator for the inverter of the proposed system against the reference current

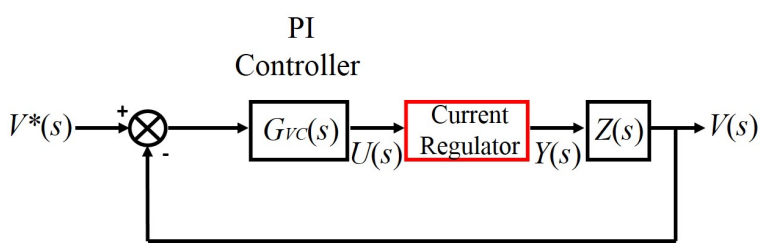

Fig. 4. Average value model diagram of the current and voltage control loops suggested for the proposed UPS system

employed in series with the controller to model the transport delay. The average-value model block diagram representation of the for the inverter of the proposed system, along with the effects of sampling and transport delay, is shown in Fig. 3(a), while a comparative analysis of the experimental response of the same current regulator against the reference current is displayed in Fig. 3(b). It is evident that the magnitude and phase difference of the response delay associated with the controller are negligible when compared to the reference current.

The proposed UPS topology is designed for extremely delicate loads whose current remains constant. As such, the reference current for any specific load will always be the same. However, if an additional load is added into the system, the reference current must be modified accordingly. This can be achieved through the use of an outer voltage loop in which the voltage error sets the current command of the inner current loop via a PI controller. The averagevalue model block diagram representation for such a system is shown in Fig. 4. As seen from the figure, a feedback voltage $V(s)$ is matched with the commanded voltage $V^{*}(s)$ to generate an error signal for the PI controller $G_{V C}(s)$. This controlled voltage error sets the current command $U(s)$ for the current regulator discussed above.

\section{Experimental Results}

To confirm the performance of the proposed UPS system, an experimental setup was constructed. The parameters of the system are:

Utility/grid: $110 \mathrm{~V}, 50 \mathrm{~Hz}$

UPS system inverter: $110 \mathrm{~V}, 50 \mathrm{~Hz}$, switching frequency $20 \mathrm{kHz}$

DC bus voltage: $176 \mathrm{~V}$

Transformer: $500 \mathrm{VA}, 110 / 110 \mathrm{~V}$

RL load: R of 5 ohms, L of $66 \mathrm{mH}$

Filter inductance $\left(L_{f}\right): 0.15 \mathrm{mH}$

A photograph of the experimental setup used to support the suggested topology is shown in Fig. 5. The hardware comprises of a DSP controller to fulfill the control requirements of the system, and power components to handle power flows. A Texas Instruments TMS320F28335 DSP with an external clock of $150 \mathrm{MHz}$ was used for processing of the data. The 12-bit ADC channel from this DSP controller was employed for load current signals coming from the current sensor installed on the load side. A carrier frequency of $20 \mathrm{kHz}$ was used for the production of PWM signals. These signals were then used by the inverter to produce single-phase output at $50 \mathrm{~Hz}$. A PI-type regulator was implemented using a TMS320F28335 DSP so as to maintain a suitable load current, while a SKM75GB128D from Semikron International was utilized to power IGBTs for the inverter power devices. A programmable single-phase ES 2000S power supply was used as a power source.

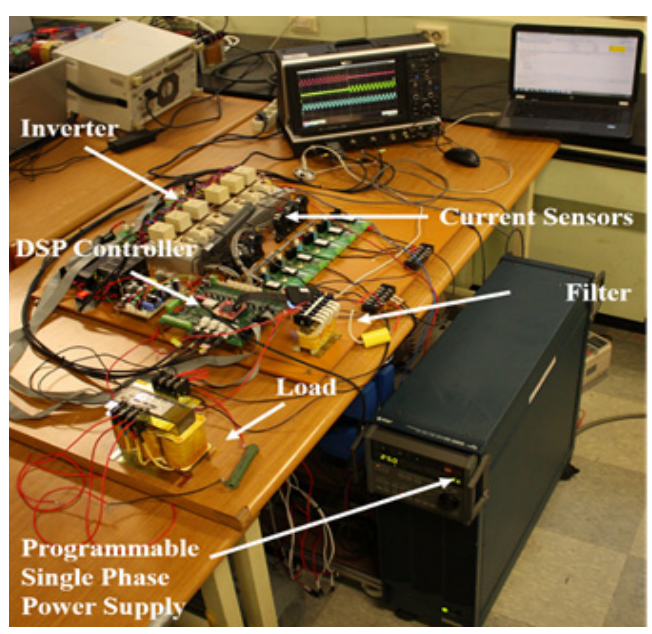

Fig. 5. Experimental setup for the proposed single-phase line-interactive UPS system 
The experimental results obtained for the suggested UPS system are presented with the following defined notations:

$\begin{array}{ll}\text { Iload } & \text { Load current } \\ \text { Iutility } & \text { Current from the utility side } \\ \text { Iinverter } & \text { Inverter current }\end{array}$

The performance of the proposed single-phase UPS system was investigated during all three possible operating modes associated with conventional UPS systems, i.e., normal operating mode, compensation mode, and inverter mode.

\section{Case 1: Normal Operating Mode}

During normal operation, the load is delivered with current from the utility, and the controller monitors the load current. Furthermore, if the UPS system is operating in inverter mode and the utility again achieves its normal power condition at a particular moment, e.g., $t=t_{0}$, the bypass switch becomes closed and the load is supplied with current from the utility. As the utility is capable of providing the rated load current (Iload), the inverter current (Iinverter) starts decreasing. During this transition of the load from the inverter to the utility, the load current (Iload) remains balanced and never surpasses the rated value. This is apparent in Fig. 6, which shows the utility, inverter, and load currents during the transition of load from the inverter to the utility using the proposed UPS system.

\section{Case 2: Compensation Mode}

Under normal conditions, the utility may suddenly experience a sag or fault. When such an event occurs, the utility supplies a reduced percentage of the rated load current. During this mode of operation, the inverter does not take over the load completely, but instead injects only a proportion of the load current along with the utility so as to avoid any unbalance. Fig. 7 shows the experimental waveforms obtained for the proposed single-phase lineinteractive UPS system as soon as the utility undergoes a

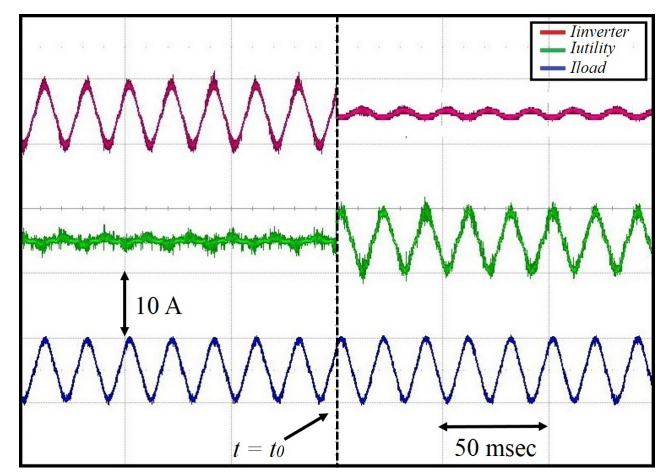

Fig. 6. Experimental results for the inverter, utility, and load currents for the proposed UPS system during normal operating mode

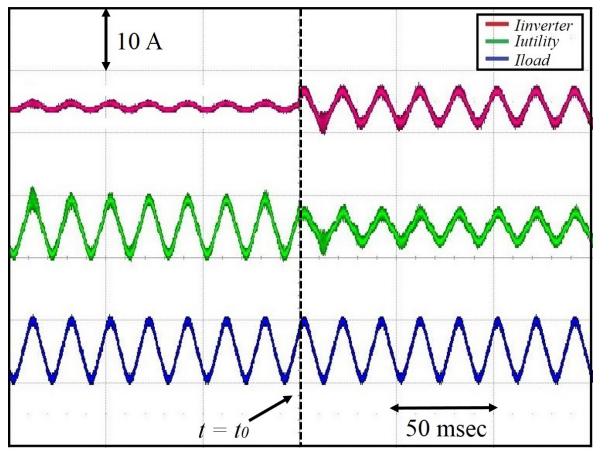

Fig. 7. Experimental results for the inverter, utility, and load currents for the proposed UPS system during compensation mode

$40 \%$ current loss during normal operation. Utility current was reduced by $40 \%$ at $t=t_{0}$ such that the inverter shared $40 \%$ of the load current with the utility. However, the load does not experience any current transients during this process.

\section{Case 3: Inverter Operating Mode}

To examine the performance of the suggested singlephase line-interactive UPS system during transition of the load from the utility to the inverter, i.e., inverter mode, experiments were performed using loading conditions similar to those employed during the normal operating and compensation modes. In the case of a blackout, the bypass switch becomes open and the load is provided power through the inverter. The process of transferring the load from the utility to the inverter is performed entirely by the high-bandwidth current control algorithm, as discussed earlier. For experimental verification of the seamless load current transition from utility to inverter mode, utility current was reduced to zero at $t=t_{0}$; the resulting waveforms of the utility, inverter, and load currents during this transition are shown in Fig. 8. As evident in the figure, the inverter takes over the load currents with no inrush currents during the change from utility to inverter.

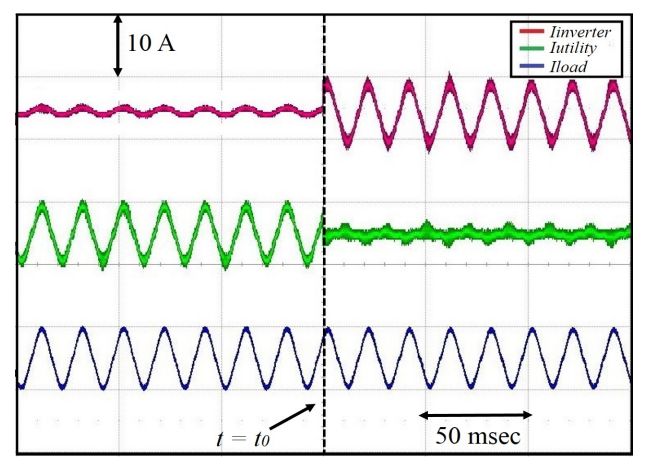

Fig. 8. Experimental results for the inverter, utility, and load currents for the proposed UPS system during inverter mode 


\section{Conclusion}

In this work, a cost-effective single-phase lineinteractive UPS system was proposed to eliminate inrush currents while powering transformer-coupled loads. A current-regulated inverter connected in parallel with the transformer and utility was employed to obtain seamless compensation of load currents and transition of the load from the utility to the inverter. It was shown that the proposed UPS system offers many advantages over conventional line-interactive UPS topologies. The operation of the suggested line-interactive UPS system during all operating modes was verified by experimental results.

\section{Acknowledgements}

This research was jointly supported by the "BK21PLUS program" through the National Research Foundation of Korea funded by the Ministry of Education and the "Human Resources Program" in Energy Technology of the Korea Institute of Energy Technology Evaluation and Planning (KETEP), granted financial resource from the Ministry of Trade, Industry and Energy, Republic of Korea. (20154030200730)

\section{References}

[1] Yu-Hsing Chen and Po-Tai Cheng, "An inrush current mitigation technique for the line-interactive uninterruptible power supply systems," IEEE Trans. on Industrial Applications, vol. 46, no. 4, July/August 2010.

[2] Bong-Hwan Kwon; Jin-Ha Choi; Tae-Won Kim, "Improved single-phase line-interactive UPS," IEEE Transactions on Industrial Electronics, vol.48, no.4, pp.804,811, Aug 2001.

[3] Bukhari, S.S.H.; Lipo, T.A; Byung-il Kwon, "An inrush current reduction technique for the lineinteractive uninterruptible power supply systems," 39th Annual Conference of the IEEE Industrial Electronics Society, vol., no., pp. 430, 434, 10-13 Nov. 2013.

[4] Yeh, C.-C.; Manjrekar, M.D., "A Reconfigurable Uninterruptible Power Supply System for Multiple Power Quality Applications," IEEE Transactions on Power Electronics, vol. 22, no. 4, pp. 1361-1372, July 2007.

[5] Wu, J. C.; Jou, H. L., “A new UPS scheme provides harmonic suppression and input power factor correction," IEEE Transactions on Industrial Electronics., vol. 42, no. 6, pp. 629-635, December 1995.

[6] Martinez, S.; Castro, M.; Antoranz, R.; Aldana, F., "Off-line uninterruptible power supply with zero transfer time using integrated magnetics," IEEE
Transactions on Power Electronics, vol. 36, no. 3, pp. 441-445, August 1989.

[7] Mokhtari, H.; Iravani, M.R.; Dewan, S.B., "Transient behavior of load transformer during sub-cycle bus transfer," IEEE Transactions on Power Electronics, vol. 18, no. 4, pp. 1342-1349, October 2003.

[8] Li, Y.; Vilathgamuwa, D. M.; Loh, P. C., "Microgrid power quality enhancement using a three-phase fourwire grid-interfacing compensator," IEEE Transactions on Industry Applications., vol. 41, no. 6, pp. 1707-1719, November/December 2005.

[9] Lee, W.-C.; Lee, T.-K.; Hyun, D.-S., “A three-phase parallel active power filter operating with PCC voltage compensation with consideration for an unbalanced load," IEEE Transactions on Power Electronics, vol. 17, no. 5, pp. 807-814, September 2002.

[10] Kong, W.Y.; Holmes, D.G.; McGrath, B.P., "Improved Stationary Frame AC Current Regulation using Feedforward Compensation of the Load EMF," 24th Annual IEEE Applied Power Electronics Conference and Exposition (APEC), vol., no., pp. 145151, 15-19 Feburary 2009.

[11] Holmes, D.G.; Lipo, T.A.; McGrath, B.P.; Kong, W.Y., "Optimized Design of Stationary Frame Three Phase AC Current Regulators," IEEE Transactions on Power Electronics, vol. 24, no. 11, pp. 2417, 2426, Nov. 2009.

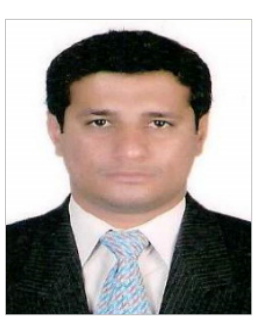

Syed Sabir Hussain Bukhari He was born in Khairpur Mir's, Sindh, Pakistan, in 1986. He received his B.E degree in Electrical Engineering from Mehran University of Engineering and Technology Jamshoro, Pakistan, in 2009. He is currently a Ph.D student in the Department of Electronic Systems Engineering, at Hanyang University, Ansan, Korea. His main research interests include electric machine design, power electronics and drive controls.

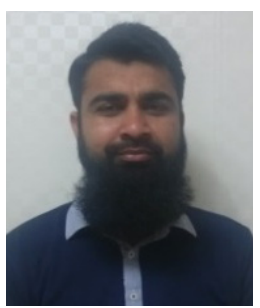

Shahid Atiq He was born in Punjab Pakistan, in 1983, He received his B.E and M.S in 2007 and 2011 respectively from University of Engineering and Technology Taxila, Pakistan. He worked in the capacity of lab engineer as well as lecturer at university of Wah and The University of Faisalabad, Pakistan. Currently he is working towards his Ph.D degree, in the Department of Electronic Systems Engineering at Hanyang University, Ansan, South Korea. His research interests include Electric Machine drives, control, and power electronics. 


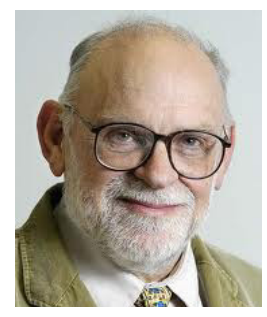

Thomas A. Lipo He was born in Milwaukee, WI, in 1938. He received his B.E.E. and M.S.E.E. from Marquette University, Milwaukee, in 1962 and 1964, respectively, and his Ph.D. in electrical engineering from the University of Wisconsin, Madison, in 1968. From 1969 to 1979 , he was an electrical engineer with the Power Electronics Laboratory, Cooperate Research and Development, General Electric Company, Schenectady, NY. In 1979, he joined Purdue University, West Lafayette, IN, as a professor of electrical engineering. In 1981, he joined the Department of Electrical and Computer Engineering, University of Wisconsin, as a professor. He has been an Emeritus Professor since January 1, 2009. He has published over 550 technical papers, 18 books and chapters, and 47 patents and has received numerous awards for his work.

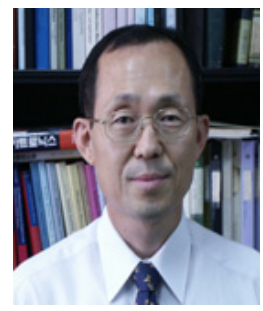

Byung-il Kwon He was born in 1956. He received his B.S. and M.S. in electrical engineering from Hanyang University, Ansan, Korea, and his Ph.D. in electrical engineering from the University of Tokyo, Tokyo, Japan, in 1989. He was a visiting researcher with the Faculty of Science and Engineering Laboratory, University of Waseda, Tokyo, from 1989 to 2000; a researcher with the Toshiba System Laboratory in 1990; a senior researcher with the Institute of Machinery and Materials Magnetic Train Business in 1991; and a visiting professor with the University of WisconsinMadison, from 2001 to 2002 . He is currently a professor at Hanyang University. His research interests are design and control of electric machines. 